\title{
Successful Treatment of Amyloid Light-chain Amyloidosis in a Charcot-Marie-Tooth Disease Patient with Lenalidomide, Cyclophosphamide, and Dexamethasone
}

\author{
Yoshitaka Kikukawa ${ }^{1}$, Hiroyuki $\mathrm{Hata}^{2}$, Mitsuharu Ueda ${ }^{3}$, Taro Yamashita ${ }^{3}$, Singo Nasu ${ }^{1}$, \\ Kazuhiko Ide ${ }^{1}$, Shikiko Ueno ${ }^{1}$, Yukio Ando ${ }^{3}$, Hiroaki Mitsuya ${ }^{1}$ and Yutaka Okuno ${ }^{1}$
}

\begin{abstract}
A 70-year-old woman with Charcot-Marie-Tooth disease (CMT) suffered from nephrotic syndrome and a renal biopsy revealed non-AA amyloid depositions that contained immunoglobulin light chain $\lambda$. Her serum $\lambda$ free LC was elevated to $80.8 \mathrm{mg} / \mathrm{L}$ and she was diagnosed with primary amyloid light-chain (AL) amyloidosis. She was subsequently treated with lenalidomide, cyclophosphamide, and dexamethasone (RCD). After 14 cycles of RCD, she achieved complete remission. Her serum albumin levels gradually normalized to $3.1 \mathrm{~g} / \mathrm{dL}$. No exacerbation of neurologic symptoms related to CMT was observed. Thus, RCD may be a well-tolerated and effective regimen for treating AL amyloidosis in patients with CMT disease.
\end{abstract}

Key words: AL amyloidosis, lenalidomide, RCD, Charcot-Marie-Tooth, neurotoxicity

(Intern Med 55: 2707-2712, 2016)

(DOI: 10.2169/internalmedicine.55.5815)

\section{Introduction}

Systemic amyloid light-chain (AL) amyloidosis is a rare disease characterized by amyloid deposits in the whole body, complicating monoclonal gammopathy of undetermined significance (MGUS), multiple myeloma, and Waldenström's macroglobulinemia. In MGUS and multiple myeloma, abnormal plasma cells produce immunoglobulin (Ig) light chain (LC), which becomes deposited in several organs, resulting in organ damage to the heart, kidney, liver, peripheral nerves, autonomic nerves, and many other organs. Cardiac amyloidosis is associated with a poor prognosis, with a mean overall survival of $<1$ year (1). Renal amyloidosis results in nephrotic syndrome, leading to severe edema, pleural and peritoneal effusions, and finally to renal failure requiring hemodialysis. The prognosis of AL amyloidosis in the absence of treatment is generally poor, and the response to conventional therapy (i.e., melphalan and dexamethasone combination therapy) is also poor. High-dose melphalan with autologous peripheral blood hematopoietic stem cell transplant (PBSCT) has demonstrated a remission rate of approximately $50 \%(2,3)$, however, patients older than 70 years of age or those with severe organ damage are ineligible for such treatment. Indeed, many patients with $\mathrm{AL}$ amyloidosis already have severe organ damage, including heart damage, at the diagnosis (4).

Several novel agents, including proteasome inhibitors bortezomib and carfilzomib and immunomodulatory drugs thalidomide, lenalidomide, and pomalidomide, have recently become available for the treatment of multiple myeloma. These drugs can dramatically improve the prognosis and prolong the survival of myeloma patients. The three-drug combination of bortezomib, cyclophosphamide, and dexamethasone (CyBorD) has been reported to be highly effective in patients with AL amyloidosis (5-7). Mikhael et al. showed that approximately $70 \%$ of patients achieved complete remission (CR), with an overall response rate of 94\% (6). We also previously found that CyBorD therapy resulted in CR in four, very good partial remission (VGPR) in

\footnotetext{
${ }^{1}$ Departments of Hematology, Rheumatology, and Infectious Disease, Kumamoto University Graduate School of Medicine, Japan, ${ }^{2}$ Division of Informative Clinical Sciences, Faculty of Medical Sciences, Kumamoto University, Japan and ${ }^{3}$ Diagnostic Unit for Amyloidosis, Department of Neurology, Kumamoto University Hospital, Japan

Received for publication May 22, 2015; Accepted for publication February 1, 2016

Correspondence to Dr. Yutaka Okuno, yokuno@gpo.kumamoto-u.ac.jp
} 
Table 1. Laboratory Data on Admission.

\begin{tabular}{|c|c|c|c|c|c|}
\hline WBC & $5,500 / \mu \mathrm{L}$ & TP & $5.0 \mathrm{~g} / \mathrm{dL}$ & T-CHO & $223 \mathrm{mg} / \mathrm{dL}$ \\
\hline $\mathrm{Hgb}$ & $14.0 \mathrm{~g} / \mathrm{dL}$ & Alb & $1.7 \mathrm{~g} / \mathrm{dL}$ & TG & $137 \mathrm{mg} / \mathrm{dL}$ \\
\hline Hct & $43.4 \%$ & $\mathrm{Na}$ & $143 \mathrm{mEq} / \mathrm{L}$ & HDL-C & $51 \mathrm{mg} / \mathrm{dL}$ \\
\hline PLT & $281 \times 10^{3} / \mu \mathrm{L}$ & $\mathrm{K}$ & $4.7 \mathrm{mEq} / \mathrm{L}$ & LDL-C & $145 \mathrm{mg} / \mathrm{dL}$ \\
\hline PT & $10.8 \mathrm{sec}$ & $\mathrm{Cl}$ & $113 \mathrm{mEq} / \mathrm{L}$ & TSH & $2.43 \mu \mathrm{U} / \mathrm{mL}$ \\
\hline PT (INR) & 0.91 & BUN & $12.3 \mathrm{mg} / \mathrm{dL}$ & BNP & $45.2 \mathrm{pg} / \mathrm{mL}$ \\
\hline APTT & $41.9 \mathrm{sec}$ & Crea & $0.50 \mathrm{mg} / \mathrm{dL}$ & $\mathrm{IgG}$ & $622 \mathrm{mg} / \mathrm{dL}$ \\
\hline Fib & $\geq 700 \mathrm{mg} / \mathrm{dL}$ & eGFR & $89 \mathrm{~mL} / \mathrm{min} / \mathrm{m}^{2}$ & $\operatorname{IgA}$ & $415 \mathrm{mg} / \mathrm{dL}$ \\
\hline ATIII & $97 \%$ & T-Bil & $0.4 \mathrm{mg} / \mathrm{dL}$ & IgM & $241 \mathrm{mg} / \mathrm{dL}$ \\
\hline$\alpha 2-\mathrm{PI}$ & $98 \%$ & AST & $29 \mathrm{U} / \mathrm{L}$ & Free $\kappa$ chain & $20.9 \mathrm{mg} / \mathrm{dL}$ \\
\hline P-FDP & $6.6 \mu \mathrm{g} / \mathrm{mL}$ & ALT & $15 \mathrm{U} / \mathrm{L}$ & Free $\lambda$ chain & $80.8 \mathrm{mg} / \mathrm{dL}$ \\
\hline D-dimer & $1.2 \mu \mathrm{g} / \mathrm{mL}$ & LD & $280 \mathrm{U} / \mathrm{L}$ & $\kappa / \lambda$ ratio & 0.261 \\
\hline TAT & $1.24 \mathrm{ng} / \mathrm{mL}$ & ALP & $499 \mathrm{U} / \mathrm{L}$ & d-FLC & $59.9 \mathrm{mg} / \mathrm{dL}$ \\
\hline \multirow[t]{2}{*}{ PIC } & $2.12 \mu \mathrm{g} / \mathrm{mL}$ & $\mathrm{CHE}$ & $624 \mathrm{U} / \mathrm{L}$ & & \\
\hline & & CRP & $0.40 \mathrm{mg} / \mathrm{dL}$ & & \\
\hline
\end{tabular}

ad-FLC: difference between involved and uninvolved FLCs

two, and partial remission in two of eight patients, with an overall response rate of $100 \%$ (7). The combination of lenalidomide, cyclophosphamide, and dexamethasone (RCD) was also reported to be effective in $\mathrm{AL}$ amyloidosis $(8,9)$. Kastritis et al. showed that RCD therapy achieved CR in $8 \%$ of patients, with an overall response rate of $55 \%$, while $\mathrm{Ku}-$ mar et al. showed that RCD therapy achieved CR and VGPR in $11 \%(4 / 35)$ and $29 \%(10 / 35)$ of patients, respectively, with an overall response rate of $60 \%$ (21/35 patients) $(8,9)$.

Charcot-Marie-Tooth disease (CMT) is a hereditary autosomal dominant disease that leads to neuralgic amyotrophy. Neuropathic drugs are therefore contraindicated in patients with CMT $(10,11)$, thus limiting potential anticancer therapies $(12,13)$. Bortezomib and thalidomide are well known to have side effects of peripheral neuropathy (14-16). We herein report a patient with AL amyloidosis and CMT who was successfully treated with RCD therapy.

\section{Case Report}

A 70-year-old Japanese woman was admitted to our hospital due to severe upper and lower edema. She had been diagnosed with CMT approximately 10 years previously. She had edema of both lower limbs 8 months before this admission, and palpebral edema of both upper limbs had exacerbated over the previous 4 months. Blood tests were performed on admission (Table 1). Her serum albumin level was $1.7 \mathrm{~g} / \mathrm{dL}$ and estimated urinary protein excretion was $6.87 \mathrm{~g} /$ day according to the urinary total protein creatinine ratio. She was diagnosed with nephrotic syndrome, and a kidney biopsy was subsequently performed (Fig. 1A). Congo red staining revealed orange-red deposits, which showed apple-green birefringence under polarized light, in the vascular walls and glomeruli. Amyloid deposits were also found in duodenum and rectum biopsy samples (Fig. 1B). These amyloid deposits were negative for staining with anti-amyloid $\mathrm{A}$, transthyretin, and $\operatorname{Ig} \operatorname{LC} \lambda$ and $\kappa$ antibodies (Fig. 2). Laser microdissection and liquid chromatography-tandem mass spectrometry revealed that the amyloid deposits contained high levels of Ig LC $\lambda$ (Table 2). $\operatorname{Ig} \mathrm{A}-\lambda$ type $\mathrm{M}$ protein was also detected by serum immunoelectrophoresis and immunofixation assays, and the free $\lambda$ chain levels were elevated $(80.8 \mathrm{mg} / \mathrm{dL})$. Plasma cells were not increased in the bone marrow $(0.9 \%)$, and the patient was therefore diagnosed with primary AL amyloidosis complicating MGUS. We concluded that her nephrotic syndrome was caused by primary AL amyloidosis.

We evaluated other potential organ damage induced by AL amyloidosis. An echocardiogram demonstrated no thickening of the ventricular wall or a granular sparkling sign, which are typical features of cardiac amyloidosis, and the serum N-terminal of the prohormone brain natriuretic peptide levels were normal (193 pg/mL), suggesting no severe heart damage. An abdominal echogram revealed mild hepatomegaly, however, her serum alkaline phosphatase levels were not highly elevated (Table 1), also indicating no severe liver damage.

We decided to treat amyloidosis-induced nephrotic syndrome with chemotherapeutic agents. However, although CyBorD therapy is effective for AL amyloidosis (5-7), bortezomib is a well-known neurotoxic agent, and it was necessary to avoid neurotoxic agents due to her coexisting CMT disease. Similarly, thalidomide is a key drug for the treatment of multiple myeloma and has demonstrated efficacy in AL amyloidosis, however, it is also a known neurotoxic agent (14-16). We therefore opted for a lenalidomidecontaining regimen. RCD therapy has previously been shown to achieve CR or VGPR in $40 \%$ of patients, with an overall response rate of $60 \%$ in patients with AL amyloidosis (9). In the previous study, the treatment schedule consisted of 4-week cycles of: lenalidomide given at $15 \mathrm{mg}$ orally (PO) on days 1-21; cyclophosphamide $300 \mathrm{mg} / \mathrm{m}^{2}$ PO given on days 1, 8, and 15; and dexamethasone $40 \mathrm{mg} \mathrm{PO}$ given on days $1,8,15$, and 22 continuously every week. Because our case was a 70-year-old patient who suffered from severe edema and CMT, we decided to reduce the doses of treatment. RCD therapy was therefore initiated with starting doses of lenalidomide $10 \mathrm{mg}$ daily for 21 days, and cyclophosphamide $300 \mathrm{mg}$ and dexamethasone $20 \mathrm{mg}$ on 
A

PAS stain
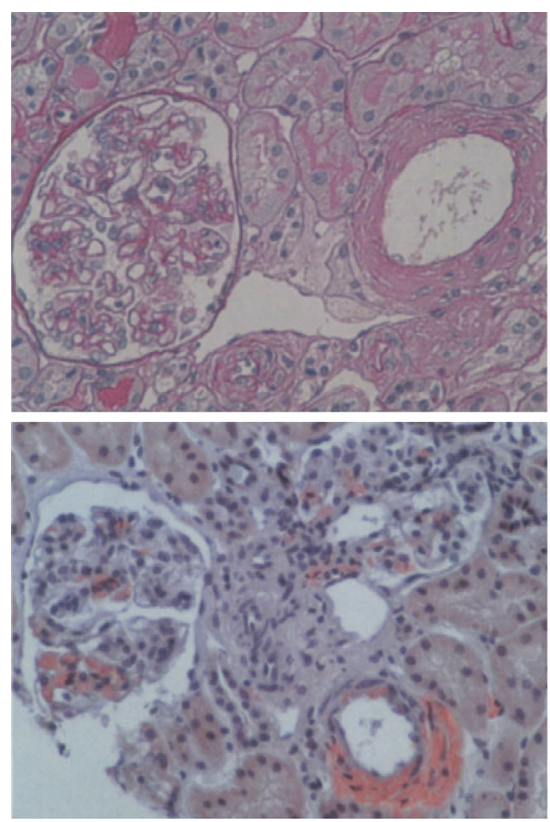

Congo red stain

B
PAS stain
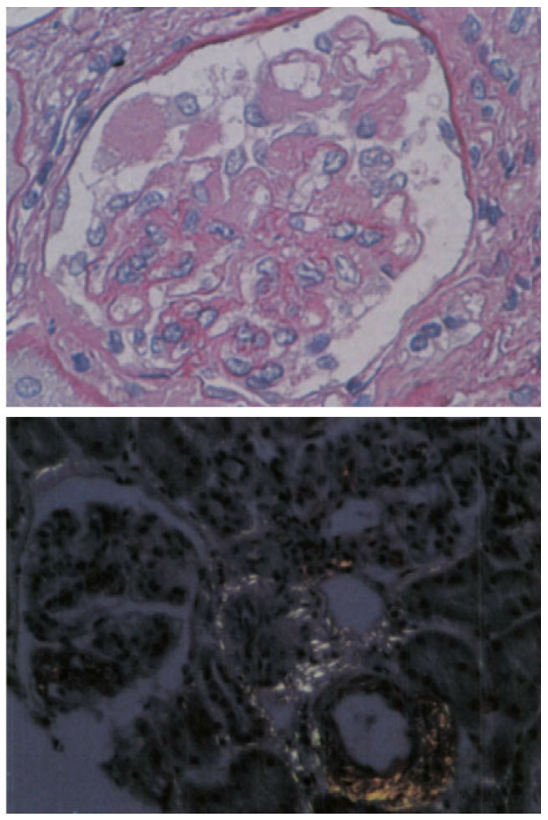

Congo red stain

(under polarized light)

\section{Endoscopic image:}

Rectum
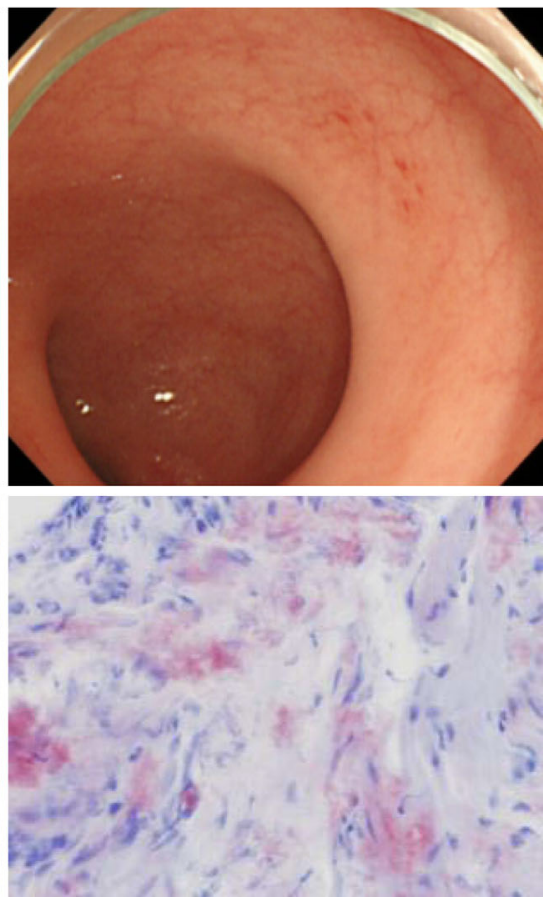

\section{Biopsy: Congo red stain}

Rectum

Figure 1. A histopathological analysis revealed non-AA amyloid deposits in the kidney and duodenal and rectal mucosae. A: A histopathological analysis of a renal biopsy sample. Renal biopsy samples showed weak periodic acid-Schiff-positive staining in the vascular walls and the glomerular mesangium (upper left and right panels). Congo red staining revealed orange-red amyloid deposits in the vascular walls and glomeruli (lower left panel). Amyloid deposits in the vascular walls and glomeruli showed apple-green birefringence under polarized light (lower right panel). B: A histopathological analysis of duodenal and rectal mucosa samples. Endoscopic images of the descending part of the duodenum (upper left panel) and rectum (upper right panel). Congo red staining revealed orange-red amyloid deposits in the duodenum (lower left panel) and the rectum (lower right panel). 
congo-red
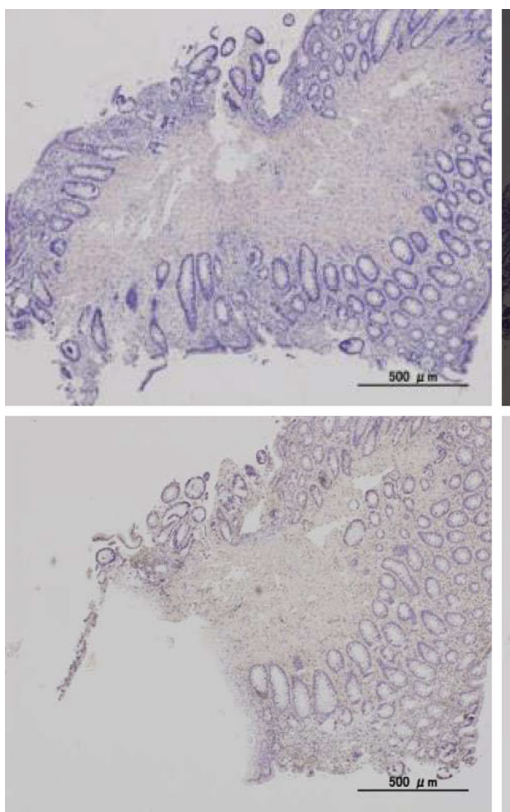

anti-serum amyloid $A$ polarized light
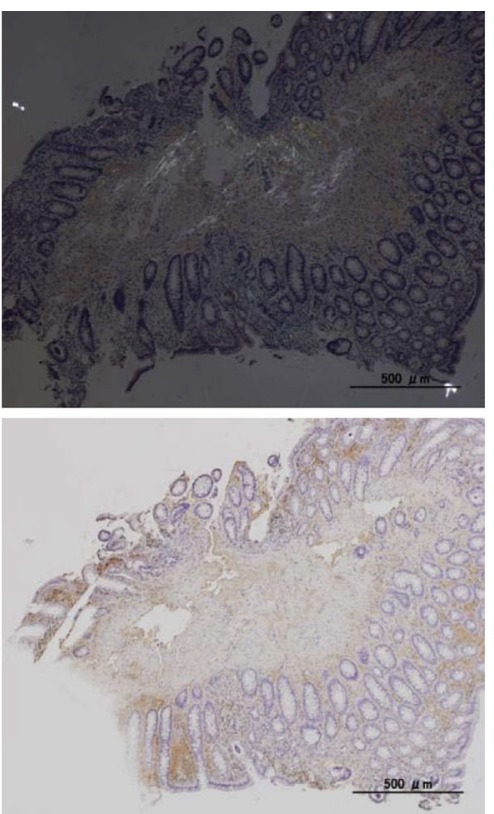

anti-IGLC $\lambda$ anti-transthyretin
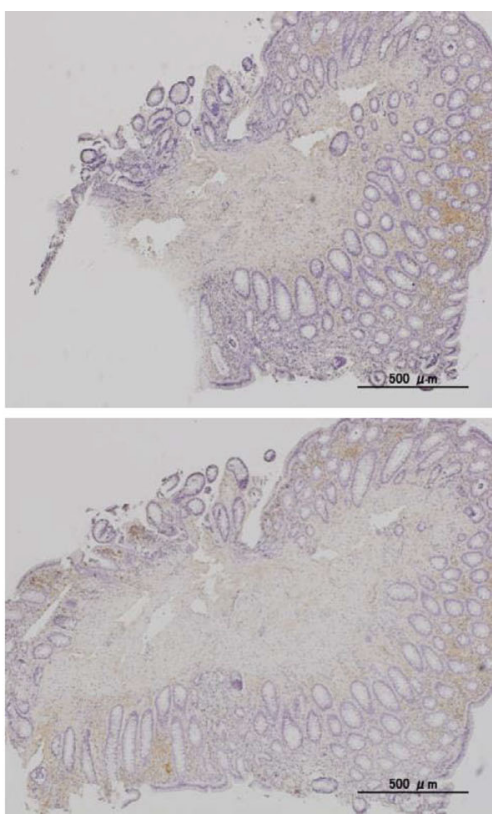

anti-IGLC $\kappa$

Figure 2. Immunohistochemical staining of amyloid deposits. AL-amyloid deposits were negative for transthyretin (upper right panel), serum amyloid A (lower left panel), Ig LC $\kappa$, and Ig LC $\lambda$ (lower middle and right panels).

Table 2. Mass Spectrometry Analysis of the Amyloid Deposits.

\begin{tabular}{llrr}
\hline Accession $^{\mathrm{a}}$ & Description & \#PSMs $^{\mathrm{b}}$ & \#AAs $^{\mathrm{c}}$ \\
\hline J00252 & Immunoglobulin lambda light chain C region (IGLC1) & 2 & 104 \\
P02649 & Apolipoprotein E & 5 & 317 \\
Q8IYA6-2 & Isoform 2 of Cytoskeleton-associated protein 2-like & 3 & 334 \\
P06702 & Protein S100-A9 & 1 & 114 \\
Q96KK5 & Histone H2A type 1-H & 1 & 128 \\
P78539-3 & Isoform 3 of Sushi repeat-containing protein SRPX & 2 & 405 \\
Q03591 & Complement factor H-related protein 1 & 1 & 330 \\
P08123 & Collagen alpha-2 (I) chain & 4 & 1,366 \\
Q9P0M6 & Core histone macro-H2A.2 & 1 & 372 \\
P06727 & Apolipoprotein A-IV & 1 & 396 \\
P22894 & Neutrophil collagenase & 1 & 467 \\
P17661 & Desmin & 1 & 470 \\
P04004 & Vitronectin & 1 & 478 \\
P15502-7 & Isoform 7 of Elastin, Homo sapiens & 1 & 677 \\
P01024 & Complement C3 & 2 & 1,663 \\
P12111-2 & Isoform 2 of Collagen alpha-3 (VI) chain, Homo sapiens & 1 & 2,971 \\
P98160 & Basement membrane-specific heparan sulfate & 1 & 4,391 \\
& proteoglycan core protein & & \\
\hline
\end{tabular}

\footnotetext{
${ }^{a}$ Accession number according to the International Nucleotide Sequence Database.

${ }^{b}$ Peptide spectrum matches. The total number of peptide spectra matched to predicted spectra according to the database.

${ }^{\mathrm{c}}$ The total number of amino acids found within peptide spectrum matches.
}

days 1,8 , and 15 . Lenalidomide was finally increased to 15 $\mathrm{mg}$ daily. Her free $\lambda$ chain level was normalized after five cycles of RCD therapy (Fig. 3). After 14 cycles of RCD therapy, her serum immunofixation test became negative for $\operatorname{IgA}-\lambda \mathrm{M}$ protein, indicating $\mathrm{CR}$. Treatment was then stopped. Her serum albumin levels gradually increased from $1.7 \mathrm{~g} / \mathrm{dL}$ to $3.1 \mathrm{~g} / \mathrm{dL}$, and her urinary protein creatinine ratio improved from 6.87 to 2.67 .
She experienced no progression of either muscle weakness or amyotrophy, and no sensory impairment. The most notable adverse event was Common Terminology Criteria for Adverse Events (CTCAE) grade 2 diarrhea, which developed during the fourth treatment cycle, requiring discontinuation of the RCD regimen for 5 weeks until diarrhea disappeared. Treatment was then restarted with lenalidomide 5 $\mathrm{mg}$ daily, gradually increased to $15 \mathrm{mg}$ daily (Fig. 3), with 


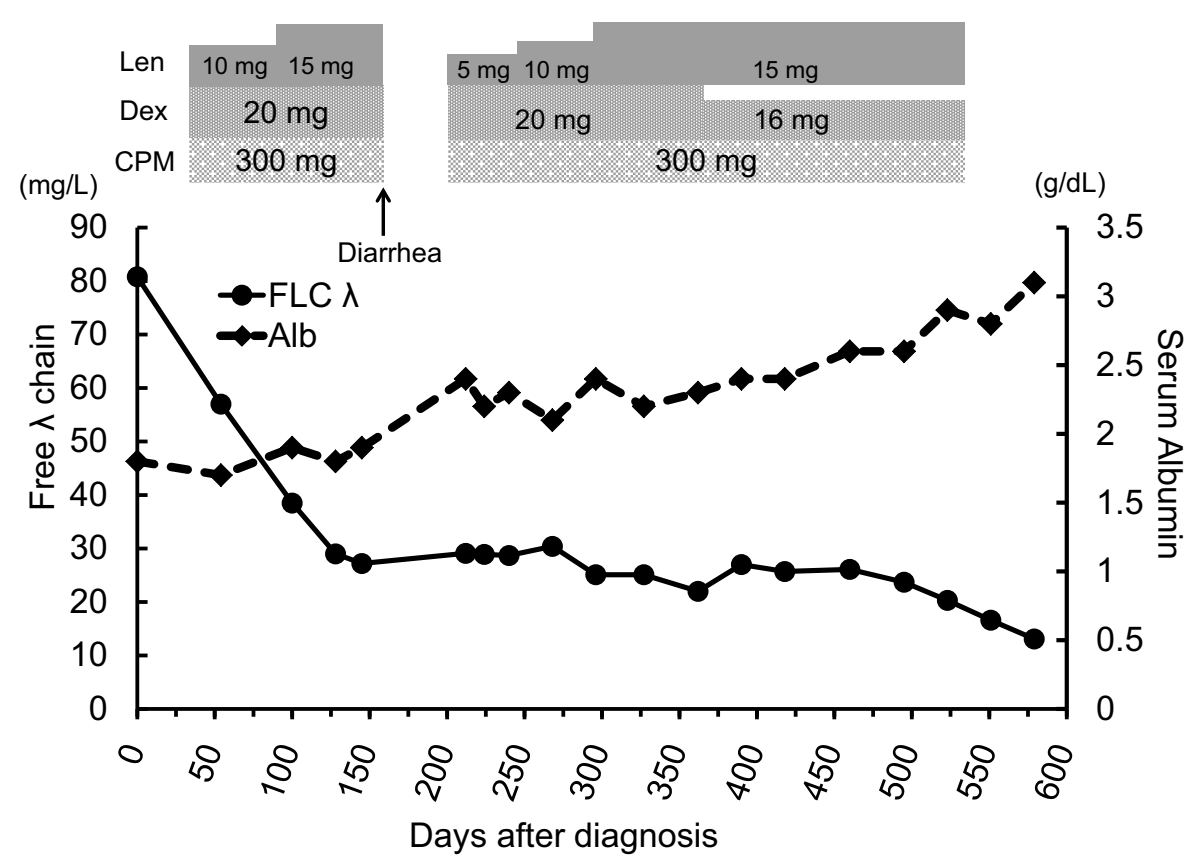

Figure 3. Clinical course of the patient. After introduction of the RCD regimen, free $\lambda$ chain was normalized to $<30 \mathrm{mg} / \mathrm{L}$ in 3 months and the serum albumin levels gradually increased to $3.1 \mathrm{~g} / \mathrm{dL}$ in 18 months. Grade 2 diarrhea appeared during the fourth cycle treatment and RCD was temporarily discontinued for 5 weeks until diarrhea resolved.

no recurrence of diarrhea. The only other adverse event was dysgeusia (CTCAE grade 1).

\section{Discussion}

We herein report the case of a 70-year-old Japanese woman with AL amyloidosis accompanying CMT disease, which was successfully treated with a RCD regimen resulting in hematological $\mathrm{CR}$ and recovery of kidney damage. There was no exacerbation of neurologic symptoms after AL amyloidosis onset in this patient, and no neuropathic progression after RCD treatment.

There are several current treatment options for AL amyloidosis. High-dose melphalan with PBSCT is an effective therapy for AL amyloidosis, however, patients with organ damage, especially heart impairment, are ineligible for this treatment (17). In addition, only approximately $50 \%$ of patients demonstrate a hematological response. Palladini et al. reported that melphalan and dexamethasone (MDex; dexamethasone $40 \mathrm{mg}$ for 4 days) was highly effective in patients with AL amyloidosis, with CR and VGPR in terms of the hematological response of $31 \%$ and $29 \%$ of the patients, respectively (18). In contrast, CR and VGPR were reduced to $12 \%$ and $20 \%$, respectively, with attenuated MDex (dexamethasone $20 \mathrm{mg}$ for 4 days). Due to the patient's age and severe edema, we had to reduce the dose of dexamethasone, and therefore, we decided not to use MDex in the current patient.

Novel drugs, including the proteasome inhibitors bortezomib and carfilzomib and the immunomodulatory drugs thalidomide, lenalidomide, and pomalidomide, have recently become available for the treatment of myeloma patients, resulting in an improvement in the 5-year survival rate from $25.6 \%$ in 1989 to $44.9 \%$ in 2005 . Regimens containing these novel agents have also been reported to be highly effective for AL amyloidosis. Kumar et al. reported that the RCD regimen was effective for AL-amyloidosis patients and obtained organ responses in eight $(31 \%)$ of 26 patients with renal involvement (9). Palladini et al. also reported a phase II prospective trial of RCD in 21 patients with refractory or relapsed $\mathrm{AL}$ amyloidosis. The hematologic response rate was $62 \%$, with one $\mathrm{CR}$ and five VGPRs. The achievement of CR/VGPR was associated with a significant survival advantage. Severe adverse events occurred in $57 \%$ of the patients, however, there were no severe neurological events and no deaths during treatment (19).

CMT is a progressive neuralgic amyotrophic disease, and more than 75 genes responsible for CMT have been identified (20). CMT has a very slow disease progression and a relatively good prognosis. However, because CMT patients tend to live longer, they may also suffer from several malignant neoplasms, in which case the potential neurotoxicity of anticancer agents becomes a matter for concern. Anticancer drugs have been reported (21) to induce severe neuralgic amyotrophy, and vincristine, one of the most neurotoxic agents, induces severe peripheral neuropathy $(10,11)$. Weimer et al. described medication-induced exacerbation of neuropathy in CMT and proposed a list of medications of concern for patients with CMT. Vincristine was listed as "Definite high risk (including asymptomatic CMT)" and bortezomib and thalidomide were also listed as "Moderate to significant risk" (21). Aging of CMT patients may also 
result in emergencies of plasma cell dyscrasia, including MGUS and multiple myeloma. Because AL amyloidosis is a complication of MGUS and multiple myeloma, aged CMT patients may also develop AL amyloidosis, as in the current case. Bortezomib is contraindicated in CMT due to its neurotoxicity, and while thalidomide is also neurotoxic, only a few reports have indicated its efficacy in AL amyloidosis $(15,16)$. We therefore chose the lenalidomide-containing regimen RCD in the current case. Five cycles of RCD therapy resulted in normalization of the free $\lambda$ chain levels, while 14 cycles induced CR, with no progression of CMT, including neuralgic amyotrophy or sensory impairment, during and after RCD therapy. RCD may thus be a safe and effective regimen for treating $\mathrm{AL}$ amyloidosis, and possibly myeloma, in patients with CMT. This case provides the first report of a patient with both CMT and AL amyloidosis and highlights the treatment options in patients with these conditions.

The authors state that they have no Conflict of Interest (COI).

\section{References}

1. Dispenzieri A, Kyle RA, Gertz MA, et al. Survival in patients with primary systemic amyloidosis and raised serum cardiac troponins. Lancet 361: 1787-1789, 2003.

2. Madan S, Kumar SK, Dispenzieri A, et al. High-dose melphalan and peripheral blood stem cell transplantation for light-chain amyloidosis with cardiac involvement. Blood 119: 1117-1122, 2012.

3. Gertz MA, Lacy MQ, Dispenzieri A, et al. Autologous stem cell transplant for immunoglobulin light chain amyloidosis: a status report. Leuk Lymphoma 51: 2181-2187, 2010.

4. Mohty D, Damy T, Cosnay P, et al. Cardiac amyloidosis: updates in diagnosis and management. Arch Cardiovasc Dis 106: 528-540, 2013.

5. Venner CP, Lane T, Foard D, et al. Cyclophosphamide, bortezomib, and dexamethasone therapy in $\mathrm{AL}$ amyloidosis is associated with high clonal response rates and prolonged progressionfree survival. Blood 119: 4387-4390, 2012.

6. Mikhael JR, Schuster SR, Jimenez-Zepeda VH, et al. Cyclophosphamide-bortezomib-dexamethasone (CyBorD) produces rapid and complete hematologic response in patients with $\mathrm{AL}$ amyloidosis. Blood 119: 4391-4394, 2012.

7. Kikukawa Y, Yuki H, Hirata S, et al. Combined use of bortezomib, cyclophosphamide, and dexamethasone induces favorable hematological and organ responses in Japanese patients with amyloid light-chain amyloidosis: a single-institution retrospective study. Int J Hematol 101: 133-139, 2015.
8. Kastritis E, Terpos E, Roussou M, et al. A phase $1 / 2$ study of lenalidomide with low-dose oral cyclophosphamide and low-dose dexamethasone (RdC) in AL amyloidosis. Blood 119: 5384-5390, 2012.

9. Kumar SK, Hayman SR, Buadi FK, et al. Lenalidomide, cyclophosphamide, and dexamethasone (CRd) for light-chain amyloidosis: long-term results from a phase 2 trial. Blood 119: 4860-4867, 2012.

10. Hildebrandt G, Holler E. Acute deterioration of Charcot-MarieTooth disease IA (CMTIA) following $2 \mathrm{mg}$ of vincristine chemotherapy. Ann Oncol 11: 743-747, 2000.

11. Nishikawa T, Kawakami K. Severe neurotoxicities in a case of Charcot-Marie-Tooth disease type 2 caused by vincristine for acute lymphoblastic leukemia. J Pediatr Hematol Oncol 30: 519-521, 2008.

12. Yerushalmi R, Levi I, Wygoda M, et al. Are platinum-based chemotherapeutic drugs safe for patients with Charcot-Marie-Tooth disease? J Peripher Nerv Syst 12: 139-141, 2007.

13. Martino MA, Miller E, Grendys EC. The administration of chemotherapy in a patient with Charcot-Marie-Tooth and ovarian cancer. Gynecol Oncol 97: 710-712, 2005.

14. Morawska M, Grzasko N, Kostyra M, Wojciechowicz J, Hus M. Therapy-related peripheral neuropathy in multiple myeloma patients. Hematol Oncol 33: 113-119, 2015.

15. Seldin DC, Choufani EB, Dember LM, et al. Tolerability and efficacy of thalidomide for the treatment of patients with light chainassociated (AL) amyloidosis. Clin Lymphoma 3: 241-246, 2003.

16. Palladini G, Perfetti V, Perlini S, et al. The combination of thalidomide and intermediate-dose dexamethasone is an effective but toxic treatment for patients with primary amyloidosis (AL). Blood 105: 2949-2951, 2005.

17. Desport E, Bridoux F, Sirac C, et al. AL Amyloidosis. Orphanet J Rare Dis 7: 1-13, 2012.

18. Palladini G, Milani P, Foli A, et al. Oral melphalan and dexamethasone grants extended survival with minimal toxicity in $\mathrm{AL}$ amyloidosis: long-term results of a risk-adapted approach. Haematologica 99: 743-750, 2014.

19. Palladini G, Russo P, Milani P, et al. A phase II trial of cyclophosphamide, lenalidomide and dexamethasone in previously treated patients with AL amyloidosis. Haematologica 98: 433-436, 2013.

20. Tazir M, Hamadouche T, Nouioua S, et al. Hereditary motor and sensory neuropathies or Charcot-Marie-Tooth diseases: An update. J Neurol Sci 347: 14-22, 2014.

21. Weimer LH, Podwall D. Medication-induced exacerbation of neuropathy in Charcot Marie Tooth disease. J Neurol Sci 242: 47-54, 2006.

The Internal Medicine is an Open Access article distributed under the Creative Commons Attribution-NonCommercial-NoDerivatives 4.0 International License. To view the details of this license, please visit (https://creativecommons.org/licenses/ by-nc-nd/4.0/).
(C) 2016 The Japanese Society of Internal Medicine http://www.naika.or.jp/imonline/index.html 